with ADRF we found that it was independent ( $p$ : 0.002) of the result of capillaroscopy, being the risk of developing the disease in a patient with positive ANA 8.5 times higher than in an ANA negative patient.

Conclusions Capillaroscopy in patients with RP has a high PNV, which allows us to estimated, with high reliability, the association of this phenomenon with autoimmune disease in patients with normal capillaroscopic patterns.

Disclosure of Interest None declared.

\section{P049 AGE-ASSOCIATED B CELLS IN EARLY DRUG-NAÏVE RHEUMATOID ARTHRITIS PATIENTS}

${ }^{1} G$ Vidal Pedrola*, ${ }^{1} \mathrm{~A}$ Pratt, ${ }^{1} \mathrm{~A}$ Mellor, ${ }^{2} \mathrm{D}$ Scheel-Toellner, ${ }^{1} \mathrm{~J}$ Isaacs, ${ }^{1} \mathrm{~A}$ Anderson. 'Inflammation, Immunology and Immunotherapy - William Leech Building, Newcastle University, Newcastle upon Tyne; ${ }^{2}$ Centre for Translational Inflammation Research, University of Birmingham, Birmingham, UK

\subsection{6/annrheumdis-2018-EWRR2019.41}

Career situation of first and presenting author Student for a master or a $\mathrm{PhD}$.

Introduction Rheumatoid arthritis (RA) is a chronic autoimmune disorder characterised by joint inflammation and bone destruction. The presence of autoantibodies, years before the clinical onset of disease, and the efficacy of Rituximab, a Bcell depleting therapy, highlight a pathogenic role for B cells. Different groups have recently identified a novel subset of $\mathrm{B}$ cells named age-associated B cells (ABCs). Studies in mice autoimmune models and patients suffering from autoimmune diseases described these cells as CD $19^{\text {high }} \mathrm{CD} 21^{-} \mathrm{CD} 11 \mathrm{c}^{+}$. Moreover, a subset of synovial fluid B cells with low levels of CD21, expresses FcRL4 and produces the cytokine RANKL, which stimulates the differentiation and activation of osteoclasts. The ABCs found in peripheral blood could therefore be the precursors of this FcRL4 positive subset found in synovia. Objectives We aimed to investigate the proportion and phenotype of peripheral blood $\mathrm{ABCs}$ in patients suffering from early drug naïve RA.

Methods Newly presenting patients, naïve to immunomodulatory treatment, were recruited from the Newcastle Early Arthritis Clinic, and followed until diagnoses were confirmed. B-cell subsets in peripheral blood were detected and phenotyped using flow cytometry. NanoString nCounter Immunlogy v2 Panel (NanoString Technologies) was used to detect mRNA from cell lysates of sorted ABCs, naïve, memory and CD5+ B cells from RA patients, age-matched healthy controls and disease controls (Psoriatic Arthritis patients).

Results Our work showed that there are no significant differences in the frequency of ABCs between RA patients, disease controls and age-matched healthy controls. There is a possible trend for increased frequencies of ABCs with age as well as those who have a high disease activity. Our results also show that $\mathrm{ABCs}$ resemble a memory $\mathrm{B}$ cells phenotype with regard to class-switch immunoglobulins expression, with a significant percentage of them being positive for $\operatorname{IgG}$ and $\operatorname{IgA}$. Interestingly, the $\mathrm{FcRL}_{4}^{+}$, the proliferating $\mathrm{Ki}^{+} 7^{+}$and the T-bet expressing $\mathrm{B}$ cells were enriched in the $\mathrm{ABC}$ population compared to the other $\mathrm{B}$ cell subsets. Furthermore, ABCs expressed high levels of MHC class II and co-stimulatory molecules, as well as the activation marker, CD69. In addition, gene expression analysis using NanoString showed differentially expressed genes between $\mathrm{ABCs}$ and memory B cells.
Conclusions These data supports an activated phenotype of the ABCs, which supports the idea that ABCs have a pathogenic role in RA, potentially via autoantibody and $\mathrm{T}$ cell stimulatory ability. However, further characterisation of this subset and functional studies are needed.

Disclosure of Interest None declared.

\section{P050 JAN GÖSTA WALDENSTRÖM AND RHEUMATOLOGY}

F Wollheim*. Department of Clinical Sciences Lund, Rheumatology. Medical Faculty, Lund University, Lund, Sweden

\subsection{6/annrheumdis-2018-EWRR2019.42}

Career situation of first and presenting author Post-doctoral fellow.

Introduction On 2. September 1943 Jan Waldenström (19061996) successfully submitted a paper to Acta Medica Scandinavica describing two patients with a new disease. The discovery was to make him world famous. ${ }^{1}$ This year marks the 75 th anniversary of macroglobulinemia and it coincided with the 10th biennial international workshop of Waldenström's macroglobulinemia, discussing advances in the genetic basis, pathogenesis and treatments of the disease. ${ }^{2}$ Jan Waldenström 7[MO1] (JW) would have enjoyed this workshop immensely, sharing the information that over $95 \%$ of the patients had somatic mutations affecting the MYD88 gene on the 2nd chromosome as well as the impressive advances in treatment. Attending this excellent meeting brought back memories of my time as Waldenström's $\mathrm{PhD}$ student and triggers me to compose this vignette, focusing on connections between the interests of my mentor and rheumatology. For a more comprehensive account of Jan Waldenström's legacy I recommend Robert Kyle's superb obituary, published in Blood, a journal JW was attached to from its start. ${ }^{3}$

After the successful defence of his landmark PhD Thesis on acute intermittent porphyria ${ }^{4}$ Waldenström's interest focused on haematology. He worked in Uppsala, a university where the study of proteins had prominence. There The Svedberg had developed the ultracentrifuge and Arne Tiselius the free electrophoresis and Robin Fåhreus the ESR. Conditions with elevated sedimentation rate (ESR) caught his special attention. An early witness is a paper from 1937, analysing 5 cases diagnosed with uveoparotitis, a rare condition most prevalent in women and considered by many to be a form of tuberculosis. He noticed the several multiorgan manifestations, the similarities with von Mikulits' disease and Boeck's sarcoid, the presence of high ESR, unspecific Wasserman reaction, absence of proof of tuberculosis, presence of xerostomia, and frequent CNS manifestations. ${ }^{5}$ Today the diagnosis could have been IgG4 related disease in several of these patients. This early paper also shows a keen interest in inflammatory systemic conditions.

In the early $1940 \mathrm{~s}$ JW collected serum from some 100 patients with longstanding ESR exceeding $120 \mathrm{~mm}$, and had it analysed by the new technique of free boundary electrophoresis by K.O. Pedersen in the Department of Physical Chemistry. In 1943 he described 3 cases from this population characterised by repeated bouts of declive purpura, leaving spots of brown discoloration, mild anaemia, and on the whole, good general health. Two of the 3 women also had dry eye problems and one had dry mouth and swollen parotis glands. $\mathrm{He}$ named the condition 'purpura 